\title{
Use of Anti-Epileptic Drugs During Adjuvant Chemo- Radiotherapy is Associated with Increased Mortality in Patients with Glioblastoma: A Nationwide Population-Based Cohort Study
}

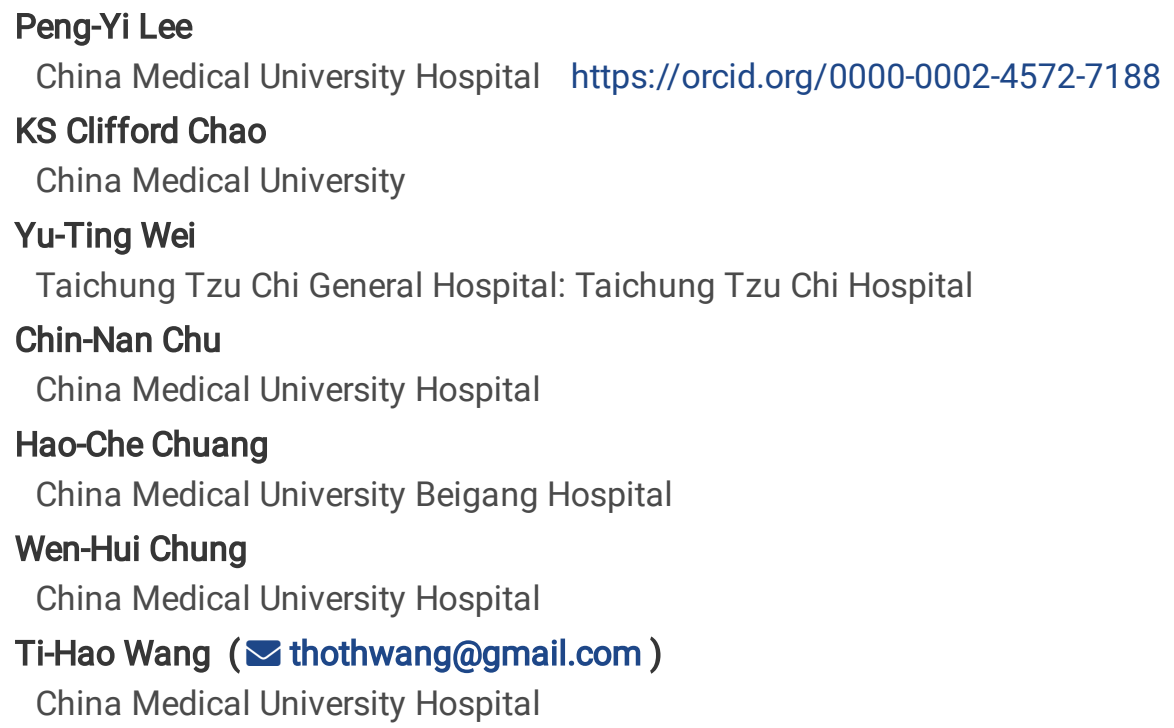

Research article

Keywords: glioblastoma, anti-epileptic drugs, radio-sensitizers, population-based study

Posted Date: December 10th, 2020

DOl: https://doi.org/10.21203/rs.3.rs-122691/v1

License: (c) (i) This work is licensed under a Creative Commons Attribution 4.0 International License. Read Full License 


\section{Abstract}

\section{Background}

There are emerging but inconsistent evidences about anti-epileptic drugs (AEDs) as radiosensitizers to improve survival in glioblastoma (GBM) patients. We conducted a population-based study to evaluate the impact of concurrent AED during postoperative chemo-radiotherapy (CCRT) on outcome.

\section{Methods}

1057 GBM patients were identified by National Health Insurance Research Database and Cancer Registry in 2008-2015. Eligible criteria included those receiving surgery, adjuvant RT and temozolomide (TMZ), and without other cancer diagnoses. Survival between patients taking concurrent AED for 14 days or more during CCRT (AED group) and those who did not (non-AED group) were compared, and subgroup analyses for valproic acid (VPA), levetiracetam (LEV), and phenytoin were performed. Multivariate analyses were used to adjust for confounding factors.

\section{Results}

There were 642 patients in the AED group while 415 in the non-AED group. The demographic data was balanced except trend of more patients in the AED group had previous drug history of AEDs. (22.6\% vs $18 \%, p=0.078)$. Overall, the AED group had significantly increased risk of mortality $(H R=1.18, p=0.016)$. In subgroup analyses, the significant detrimental effect was demonstrated in VPA (HR=1.29, $p=0.0002)$, but not in LEV $(H R=1.18, p=0.079)$ and phenytoin $(H R=0.98, p=0.862)$. When stratified by sex and age, this detrimental effect was seen in male and patients less than 65 years old in the VPA group.

\section{Conclusion:}

Survival was not improved and even worse in patients with AEDs during CCRT in this nationwide population-based study. Our real-world data did not support prophylactic use of AEDs for GBM.

\section{Background}

The glioblastoma multiforme (GBM) is the most common primary malignant brain tumor. It is a devastating disease and almost universally fatal. The peak incidence is between 65 and 75 years of age. In the past years, the treatment paradigm has been evolving, but the result is still frustrating. Glioblastoma is not surgically curable because of its nature of extensive infiltration. In the 20th century, post-operative radiotherapy (RT) was proved to ameliorate patient survival [1-3], and the treatment field shifted from whole brain to partial brain according to studies about patterns of local failure [4-6]. Many efforts had been made trying to further improve outcome, such as alter-fractionation schedule, dose-escalation trials, concurrent chemotherapy but had little effect until the Stupp regimen being published in 2005 [7, 8]. After maximal safe resection, daily $75 \mathrm{mg} / \mathrm{m}^{2}$ temozolomide (TMZ) concomitant to fractionated RT equivalent to 60 Gray and followed by a daily TMZ dose of $150-200 \mathrm{mg} / \mathrm{m}^{2}$ for 5 days every 28 days as the subsequent maintenance chemotherapy is the standard of care nowadays. The research topic getting popular recently is about anti-epileptic drugs (AEDs), such as valproic acid (VPA), have been proposed to be radio-sensitizers [9, 10]. Eui Kyu Chie et al. founded evident in vitro and in vivo radiosensitizing effect of VPA by the method of tumor growth delay [9]. Dinesh Thotala et al pointed out VPA not only enhanced the efficiency of glioma RT but also mitigated neurocognitive deficits by protecting hippocampal neurons [10]. But there are other reports against the claim. Marita Eckert et al concluded VPA did not impair clonogenic survival or radioresistance of primary GBM spheroid cultures treated with RT and TMZ. So they did not advocate a general use of VPA as a radiosensitizer in anti-GBM therapy [11]. Furthermore, the clinical results were also controversial. A single-arm phase II study showed impressive results with one-year overall survival (OS) and progression free survival (PFS) were $86 \%$ and $43 \%$, respectively, when VPA was added to adjuvant RT and TMZ [12]. However, a pooled analysis of prospective clinical trials presented in 2016 revealed no improvement of outcome [13]. Since there is an emerging but inconsistent body of evidence, we conducted a population-based study to investigate the impact of concurrent AEDs during postoperative chemo-radiotherapy (CCRT) on outcome in patients with GBM. 


\section{Methods}

\section{Study subjects}

We collected GBM patient data from the Cancer Registry database in Taiwan between 2008 and 2015. The data was confirmed with a Longitudinal Health Insurance Database for Catastrophic Illness Patients, a part of Taiwan National Health Insurance research databases (NHIRDs), by the code of International Classification of Diseases, Ninth and Tenth Revision, Clinical Modification (ICD-9-CM and ICD-10-CM). The NHI system was established by the Taiwanese government in 1995, which covers nearly all Taiwanese citizens. The NHIRDs contains medical claim data which included the registry of beneficiaries, disease registry profile, drug prescriptions, and other medical services. The database underwent de-identification before it was released for research use. The research ethics committee approved this study.

GBM patients with radical operation and adjuvant RT and TMZ treatment (RT/TMZ) were the study subjects. Patients with other cancer diagnoses were excluded. The AED history was also identified from the NHIRDs by the drug codes of World Health Organization/Anatomical Therapeutic Chemical Classification (WHO/ATC). Patients who had at least one AED more than 14 days during RT/TMZ were defined as the experimental group (AED group), and those who did not were in the control group (nonAED group). The three most commonly used AEDs were in order of VPA, levetiracetam (LEV), and phenytoin.

\section{Statistical analysis}

The age $(<40,40-65,>65$ years of age), sex, the year of diagnosis, Charlson comorbidity index (CCl, categorized as $0,1-2, \geq 3$ points), previous drug history of AEDs before brain tumor diagnosis, status of death till end of follow up between two cohorts were presented with the number and percentage. Chi-square test was used to test the difference between two categorical variables. Survival analyses were performed by the Kaplan-Meier method, and the log-rank test was used to evaluate the difference. Cox proportional hazards models were used to calculate hazard ratios (HRs) and 95\% confidence intervals (Cls) for whether AEDs were associated with OS. Univariate analyses were followed by multivariate analyses adjusting for potential confounding factors. A two-sided p-value $<0.05$ was considered statistically significant. All statistical analyses were performed using SAS software version 9.4 (SAS Institute, Cary, NC).

\section{Results}

A total of 1057 GBM patients with post-operative RT/TMZ were enrolled. Of them, 642 patients were in the AED group, and the other 415 were in the non-AED group. Regardless of monotherapy or polytherapy of AEDs, 406 patients were treated with VPA, 153 with LEV, 119 with phenytoin. The age distribution, sex, year of diagnosis, CCl, and status of death till end of follow up were comparable between two groups. There was a trend toward more patients in the AED group had previous drug history of AEDs before GBM diagnosis then in the non-AED group (22.6\% vs $18 \%, p=0.078)$. During the study period, $557(86.8 \%)$ and 347 (83.6\%) patients died in AED and non-AED group, respectively $(p=0.156)$. (Table 1$)$ 
Table 1

Demographic data of study population

\begin{tabular}{|c|c|c|c|c|c|}
\hline & \multicolumn{2}{|c|}{ AED group $(n=642)$} & \multicolumn{2}{|c|}{ non-AED group $(n=415)$} & \multirow[b]{2}{*}{$\mathrm{p}$-value } \\
\hline & Number (n) & $\%$ & Number (n) & $\%$ & \\
\hline Sex & & & & & 0.376 \\
\hline Male & 366 & 57 & 248 & 59.8 & \\
\hline Female & 276 & 43 & 167 & 40.2 & \\
\hline Age (year) & & & & & 0.617 \\
\hline$<40$ & 92 & 14.3 & 58 & 14 & \\
\hline $40-65$ & 382 & 59.5 & 237 & 57.1 & \\
\hline$>65$ & 168 & 26.2 & 120 & 28.9 & \\
\hline Year of diagnosis & & & & & 0.464 \\
\hline 2008 & 60 & 9.35 & 39 & 9.4 & \\
\hline 2009 & 73 & 11.37 & 30 & 7.23 & \\
\hline 2010 & 71 & 11.06 & 59 & 14.22 & \\
\hline 2011 & 77 & 11.99 & 48 & 11.57 & \\
\hline 2012 & 86 & 13.4 & 57 & 13.73 & \\
\hline 2013 & 77 & 11.99 & 53 & 12.77 & \\
\hline 2014 & 101 & 15.73 & 66 & 15.9 & \\
\hline 2015 & 97 & 15.11 & 63 & 15.18 & \\
\hline $\mathrm{CCl}$ & & & & & 0.905 \\
\hline 0 & 244 & 38 & 154 & 37.1 & \\
\hline $1-2$ & 268 & 41.7 & 179 & 43.1 & \\
\hline$\geq 3$ & 130 & 20.3 & 82 & 19.8 & \\
\hline Drug history of AED before diagnosis & 145 & 22.6 & 75 & 18.1 & 0.078 \\
\hline Status of death & 557 & 86.76 & 347 & 83.61 & 0.156 \\
\hline \multicolumn{6}{|l|}{ Type of AED a } \\
\hline VPA & 406 & & & & \\
\hline LEV & 153 & & & & \\
\hline Phenytoin & 119 & & & & \\
\hline Others & 135 & & & & \\
\hline AED monotherapy & 442 & 68.9 & & & \\
\hline VPA & 277 & & & & \\
\hline LEV & 82 & & & & \\
\hline
\end{tabular}

Abbreviations: $\mathrm{AED}=$ anti-epileptic drugs; $\mathrm{CCl}=$ Charlson comorbidity index; VPA = valproic acid $; \mathrm{LEV}=$ levetiracetam

a. The use of AED could be monotherapy or polytherapy, thus, total number exceeded total patient number. 


\begin{tabular}{|lc|}
\hline & AED group $(\mathrm{n}=\mathbf{6 4 2}) \quad$ non-AED group $(\mathrm{n}=\mathbf{4 1 5})$ \\
\hline Phenytoin & 70 \\
\hline Others & 16 \\
\hline Abbreviations: AED = anti-epileptic drugs; $C C I$ = Charlson comorbidity index; VPA = valproic acid ; LEV = levetiracetam \\
\hline a. The use of AED could be monotherapy or polytherapy, thus, total number exceeded total patient number. \\
\hline
\end{tabular}

The median survival is 17.5 months in the AED group while 21 months in the non-AED group. The 2-year and 5-year survival rates are $35.2 \%$ and $10.7 \%$ in the AED group, whereas $43.6 \%$ and $13.4 \%$ in the non-AED group, respectively. Univariate cox proportional hazards model showed higher risk of mortality in the AED group compared to the non-AED group (HR 1.22, $p=0.004)$. Among potential confounding factors, male, older age, higher comorbidity score, and previous drug history of AEDs before GBM diagnosis were associated with poorer survival. Taking more TMZ seemed to be protective (HR 0.93, $p<0.0001)$. In the subgroup analyses of patients using different types of AED, those with VPA or LEV during RT/TMZ had a significantly worse prognosis (HR 1.317, $p<0.0001$ for VPA) (HR 1.308, $p=0.004$ for LEV). Concurrent phenytoin had no effect on survival $(H R 0.935, p=0.522)$. (Table 2) (Fig. 1) 
Univariate analysis of demographics, comorbidity, and treatment-related factors for survival

\begin{tabular}{|c|c|c|c|}
\hline & HR & $95 \% \mathrm{Cl}$ & p-value \\
\hline \multicolumn{4}{|c|}{ Concurrent AED during RT $\geq 14$ days } \\
\hline AED group & 1.216 & $(1.063-1.39)$ & $0.004^{\star}$ \\
\hline Non-AED group & REF & & \\
\hline \multicolumn{4}{|c|}{ Subgroups of AED } \\
\hline VPA & 1.317 & $(1.153-1.506)$ & $<0.0001^{\star}$ \\
\hline LEV & 1.308 & $(1.087-1.574)$ & $0.004^{\star}$ \\
\hline Phenytoin & 0.935 & $(0.761-1.149)$ & 0.522 \\
\hline Non-AED group & REF & & \\
\hline \multicolumn{4}{|l|}{ Sex } \\
\hline Male & 1.303 & $(1.139-1.490)$ & $0.0001^{\star}$ \\
\hline Female & REF & & \\
\hline \multicolumn{4}{|l|}{ Age } \\
\hline$>65$ & 2.027 & $(1.621-2.535)$ & $<0.0001^{*}$ \\
\hline $40-65$ & 1.289 & $(1.049-1.584)$ & $0.016^{*}$ \\
\hline$<40$ & REF & & \\
\hline \multicolumn{4}{|c|}{ Year of diagnosis } \\
\hline 2008 & 0.898 & $(0.673-1.198)$ & 0.465 \\
\hline 2009 & 1.211 & $(0.920-1.595)$ & 0.171 \\
\hline 2010 & 0.993 & $(0.761-1.295)$ & 0.959 \\
\hline 2011 & 1.089 & $(0.836-1.420)$ & 0.528 \\
\hline 2012 & 1.113 & $(0.861-1.440)$ & 0.414 \\
\hline 2013 & 1.093 & $(0.838-1.424)$ & 0.513 \\
\hline 2014 & 1.111 & $(0.862-1.433)$ & 0.416 \\
\hline 2015 & REF & & \\
\hline \multicolumn{4}{|c|}{ Drug history of AED before diagnosis } \\
\hline Yes & 1.211 & $(1.033-1.421)$ & $0.019 *$ \\
\hline No & REF & & \\
\hline \multicolumn{4}{|l|}{$\mathrm{CCl}$} \\
\hline$\geq 3$ & 1.499 & $(1.252-1.794)$ & $<0.0001^{*}$ \\
\hline $1-2$ & 1.279 & $(1.102-1.484)$ & $0.001^{\star}$ \\
\hline 0 & REF & & \\
\hline
\end{tabular}

Abbreviations: $\mathrm{AED}=$ anti-epileptic drugs; $\mathrm{CCl}=$ Charlson comorbidity index; $\mathrm{VPA}=$ valproic acid $; \mathrm{LEV}=$ levetiracetam; $\mathrm{TMZ}=$ temozolomide 


\begin{tabular}{|c|c|c|c|}
\hline & HR & $95 \% \mathrm{Cl}$ & p-value \\
\hline \multicolumn{4}{|c|}{ TMZ dose (as continuous variable) } \\
\hline Dose (mg) & 0.929 & $(0.914-0.945)$ & $<0.0001 *$ \\
\hline
\end{tabular}

Multivariate analyses was performed to adjust for age, sex, previous drug history of AEDs, CCI score, TMZ dosage, and other AEDs in patients with polytherapy. The HR for death was still significantly higher in the AED group then in the non-AED group (HR $1.18, p=0.016)$. When stratified by sex and age, concurrent AEDs during RT/TMZ for more than 14 days was harmful in male (HR 1.3, $p=0.003)$, however, no significant effect in female (HR 1.057, $p=0.6)$. There was also no significance for patients in 3 different age groups. In subgroup analyses for patients taking the three most common drugs, the survival deteriorated in patients taking VPA compared to those who did not (HR 1.29, $p=0.0002)$. When stratified by sex and age, male and patient less than 65 years old had significantly higher risk of death if concurrent VPA was prescribed. Nevertheless, this significant detrimental effect was not observed in patients with LEV or phenytoin. Notably, the impact of LEV was statistically significant in univariate analysis, but there was an adverse trend after adjustment for other confounding factors (HR 1.182, p = 0.079) (Table 3).

Table 3

Multivariate analyses ${ }^{\mathrm{a}}$ for survival stratified by sex and age and subgroup analyses

\begin{tabular}{|c|c|c|c|c|c|c|c|c|c|c|c|c|}
\hline & \multicolumn{3}{|c|}{$\begin{array}{l}\text { AED group } \\
(n=642)\end{array}$} & \multicolumn{3}{|c|}{$\begin{array}{l}\text { VALPROIC } \\
\text { ACID }(n=406)\end{array}$} & \multicolumn{3}{|c|}{$\begin{array}{l}\text { LEVETIRACETAM } \\
(n=153)\end{array}$} & \multicolumn{3}{|c|}{$\begin{array}{l}\text { PHENYTOIN } \\
(n=119)\end{array}$} \\
\hline & $\mathrm{HR}$ & $95 \% \mathrm{Cl}$ & $\begin{array}{l}\mathrm{p}- \\
\text { value }\end{array}$ & $\mathrm{HR}$ & $95 \% \mathrm{Cl}$ & p-value & $\mathrm{HR}$ & $95 \% \mathrm{Cl}$ & $\begin{array}{l}\mathrm{p}- \\
\text { value }\end{array}$ & $\mathrm{HR}$ & $95 \% \mathrm{Cl}$ & $\begin{array}{l}\mathrm{p}- \\
\text { value }\end{array}$ \\
\hline Overall & 1.18 & $\begin{array}{l}(1.032- \\
1.354)\end{array}$ & $0.016^{\star}$ & 1.29 & $\begin{array}{l}(1.128- \\
1.484)\end{array}$ & $0.0002^{\star}$ & 1.18 & $\begin{array}{l}(0.981- \\
1.425)\end{array}$ & 0.079 & 0.98 & $\begin{array}{l}(0.792- \\
1.214)\end{array}$ & 0.86 \\
\hline \multicolumn{13}{|l|}{ Sex } \\
\hline Male & 1.3 & $\begin{array}{l}(1.096- \\
1.552)\end{array}$ & $0.003^{*}$ & 1.47 & $\begin{array}{l}(1.233- \\
1.760)\end{array}$ & $<.0001 *$ & 1.11 & $\begin{array}{l}(0.874- \\
1.405)\end{array}$ & 0.37 & 1.12 & $\begin{array}{l}(0.850- \\
1.476)\end{array}$ & 0.42 \\
\hline Female & 1.06 & $\begin{array}{l}(0.848- \\
1.316)\end{array}$ & 0.6 & 1.1 & $\begin{array}{l}(0.883- \\
1.375)\end{array}$ & 0.39 & 1.34 & $\begin{array}{l}(0.982- \\
1.826)\end{array}$ & 0.06 & 0.83 & $\begin{array}{l}(0.590- \\
1.175)\end{array}$ & 0.30 \\
\hline \multicolumn{13}{|l|}{ Age } \\
\hline$<40$ & 1.42 & $\begin{array}{l}(0.946- \\
2.132)\end{array}$ & 0.09 & 2.26 & $\begin{array}{l}(1.492- \\
3.427)\end{array}$ & $0.0001 *$ & 1.45 & $\begin{array}{l}(0.834- \\
2.506)\end{array}$ & 0.19 & 1.52 & $\begin{array}{l}(0.856- \\
2.712)\end{array}$ & 0.15 \\
\hline $40-65$ & 1.13 & $\begin{array}{l}(0.943- \\
1.347)\end{array}$ & 0.19 & 1.27 & $\begin{array}{l}(1.055- \\
1.520)\end{array}$ & $0.01 *$ & 1.22 & $\begin{array}{l}(0.944- \\
1.568)\end{array}$ & 0.13 & 0.83 & $\begin{array}{l}(0.623- \\
1.092)\end{array}$ & 0.18 \\
\hline$>65$ & 1.22 & $\begin{array}{l}(0.948- \\
1.576)\end{array}$ & 0.12 & 1.04 & $\begin{array}{l}(0.810- \\
1.347)\end{array}$ & 0.74 & 1.08 & $\begin{array}{l}(0.768- \\
1.514)\end{array}$ & 0.66 & 1.34 & $\begin{array}{l}(0.868- \\
2.069)\end{array}$ & 0.19 \\
\hline \multicolumn{13}{|c|}{ Abbreviations: AED = anti-epileptic drugs } \\
\hline
\end{tabular}

Additional sensitivity tests were conducted to clarify if the detrimental effects persisted in the AED cohort with monotherapy.

There were 442 patients receiving monotherapy. Of them, 277 patients were treated with VPA, 82 with LEV, and 70 with phenytoin. The results still supported the conclusions (Additional Table 1).

\section{Discussion}


The diagnosis of GBM confers a dismal prognosis, with a median OS of only 14 to 18 months even with standard treatment. Efforts at improving OS have had only modest success. In-field or marginal recurrences after RT are common, leading to the assumption that outcomes will be improved if we could find an agent sensitizing GBM cells to RT. Many recent in vitro and in vivo evidences has emerged indicating that AEDs may act synergistically with RT, moreover, AEDs may have anti-tumor effects themselves. VPA is the drug being discussed most frequently, and it is an antiepileptic agent with histone deacetylase inhibitor (HDACi) activity shown to sensitize GBM cells in preclinical models, to promote hyperacetylation of DNA-binding histone proteins together with decondensation of chromatin, and to induce a demethylation/activation process of tumor suppressor genes. However, these results are not universal and have been the subject of much debate.

In our study, the inferior OS was observed in the AED group and in the subgroup of VPA. This detrimental effect seemed to be evident in male and in younger patients. Regarding age, those younger than 40 years old had a trend of higher risk of death in the AED group compared to the non-AED group, while the HR for death was significantly higher for those younger than 65 years old in the subgroup of VPA. (Table 3) The underlying cause of these differences remains unclear. In the literature review, results of clinical studies are inconsistent as well. A single arm phase II study enrolled 37 patients with GBM, receiving VPA, $25 \mathrm{mg} / \mathrm{kg}$ orally, divided into 2 daily doses concurrent with RT and TMZ. It was inspiring that the median OS reached 29.6 months, and median PFS was 10.5 months [12]. A retrospective analysis of 544 patients from Memorial Sloan-Kettering Cancer center showed that VPA use during RT was associated with improved OS. Patients with AEDs for more than half the duration of RT were enrolled. Of the 5 most common AEDs during RT, only VPA was associated with significantly improved OS (HR 0.67, p = 0.047). When the analysis was restricted to those receiving concurrent TMZ, VPA use was marginally associated with better OS (HR 0.54 , $p=0.057$ ). The results implied that HDAC inhibitors, like VPA, may enhance the effect of RT and should be subjected to future clinical trials [14]. An assessment of the impact of the interaction between AED use and CCRT on survival was performed in the European Organization for Research and Treatment of Cancer/ National Cancer Institute of Canada (EORTC/ NCIC) TMZ trial, and it showed prolonged survival with VPA use [15]. Nevertheless, a combined analysis of four contemporary randomized trials with individual patient information offered a different perspective. They evaluated PFS and OS between two conditions: any VPA or LEV use and no use at baseline, or VPA or LEV use both at start of and still after CCRT. The authors concluded that VPA or LEV use was not beneficial, and did not being justified for reasons other than seizure control outside clinical trials [13].

An unresolved issue is that whether the positive effect of VPA occurs only in patients undergoing RT without TMZ? The phase II study mentioned before included a small number of patients and there was no control arm. Although all of the 37 patients planned to be treated with concomitant RT/TMZ, there were 8 patients $(21.6 \%)$ stopped TMZ mainly due to bone marrow suppression [12]. The study from MSKCC enrolled patients between 1998 and 2008, and only about one-third of this cohort (34.8\%) had taken TMZ during RT [14]. So their result must be interpreted carefully. In the pooled analysis of AVAGlio, CENTRIC, CORE, and RTOG 0825, the patient data were obtained from TMZ-containing arms of the four trials. They indicated that the OS was not improved with VPA use both at baseline (HR 0.96, $p=0.633)$ and at start of and still after CCRT $(H R 1.10, p=0.44)$ [13]. One of the advantages of our study is that all subjects received the standard treatment of concomitant RT/TMZ and adjuvant TMZ; therefore we made a greater contribution to current clinical practice compared to previous publications. The interaction between VPA and TMZ has not been fully understood. Although many retrospective series indicated VPA use might be associated with improved survival, publication bias inevitably occurred. To date, there is no randomized data to illustrate this issue. Our result discouraged the routine use of VPA during RT/TMZ.

With regard to LEV, our data showed there was a trend to be worse when LEV being prescribed during RT/TMZ (HR 1.18, $p=$ 0.079), furthermore, it seemed to be more harmful in female (HR 1.34, $p=0.06$ ). Notably, male had significantly higher risk of death in the subgroup of VPA (HR 1.47, p < 0.0001) (Table 3). Another retrospective, single-center study reported different conclusion about LEV. 418 patients were treated per the current protocol and all used at least one AED. A total of nine AEDs were evaluated, and the three most common drugs are LEV, VPA, and gabapentin. The significant benefit existed only in patients with LEV compared to those without it (median OS: 21 versus 16 months, $p<0.001$ ). To go a step further, the positive impact of LEV on OS was seen in the group with a methylated MGMT promoter (HR 0.174, $p=0.006)$, but not in the unmethylated group $(p=$ 0.623). In addition, the median OS in patients with VPA was shorter than those without VPA (18 vs 20 months, $p=0.38$ ) [16]. Although the difference was not statistically significant, the adverse effect of VPA on OS was consistent with our work. Our result still did not show the benefit of LEV during RT/TMZ. 
Likewise, the results of pre-clinical studies were also controversial. Eui Kyu Chie et al demonstrated evident radiosensitizing effect for fractionated RT of VPA in tumor bearing mice with two different cell lines [9]. Dinesh Thotala et al revealed that VPA led to significant tumor growth delay and radiosensitization with survival benefit, inhibition of cancer cell proliferation, cell cycle arrest, and accumulation at $\mathrm{G}_{2} / \mathrm{M}[10]$. Zhiying $\mathrm{Li}$ et al reported survival rate in human glioma cell populations exposed to VPA + TMZ or ACNU was significantly decreased compared with that of the TMZ or ACNU alone groups. VPA not only enhanced the inhibitory effects of $T M Z$ and $A C N U$ but also induced tumor apoptosis [17]. However, there are researchers holding the converse opinion. A study from Germany used three GBM cell lines and primary spheroid cultures to evaluate the effect of VPA, and did not suggest a radio-sensitizing effect of VPA in general at concentrations achieved in the clinical situation. They also observed VPAmediated acceleration of GBM cell migration which might boost tumor spreading and brain infiltration [11].

Another issue worthy of discussion is that the prognostic value of epilepsy in patients with GBM. An incidence of epilepsy was in the range of $25-60 \%$ in the literature. Some authors believed that epileptogenic GBM convey a favorable outcome, which might be due to early diagnosis [18-20]. But there are still other studies referring that epilepsy at presentation is not an independent prognostic factor for longer survival [21]. Additionally, Sharon Berendsen et al reported that for those who presented with epilepsy, the use of VPA did not associate with survival [18]. The aim of AED use is therapeutic or prophylactic may play a role in outcomes. Anticonvulsant prophylaxis is not recommended in patients with newly diagnosed primary or secondary brain tumors, especially in light of a significant risk of serious adverse events and problematic drug interactions. The incidence of

anticonvulsant side effects appears to be higher $(20$ to $40 \%)$ in brain tumor patients than in general population. This increment is due at least in part to the additive or synergistic effects of concurrently administered drugs (especially chemotherapeutic agents) and to the underlying brain tumors $[22,23]$. In our study population, there are more people in the AED group had the drug history of anti-convulsants before GBM diagnosis than in the non-AED group $(22.6 \%$ versus $18.1 \%, p=0.078)$, and these patients had poorer outcome (HR for death $1.211, p=0.019)$. The superiority of our study is that we adjusted for past history of AED use as a potential confounding factor of survival.

There are some disadvantages in our study. First, the genetic alterations of 06-methylguanine-DNA methyltransferase (MGMT) promoter methylation status and isocitrate dehydrogenase mutations were not available in the NHIRD. Second, other factors associated with OS such as patient performance status, Recursive Partitioning Analysis classes, and clinic-pathological parameters (eg. extent of initial resection, tumor location, and number of lesions) were not analyzed due to the limitation of database.

In order to overcome these shortcomings, we performed sensitivity analyses to simulate the distribution of IDH1 mutation and methylation status of MGMT in our cohort. Comprehensive literature review was performed, especially focusing on data in eastern countries. Approximately, the incidences of IDH1 mutation and MGMT promotor methylation are $15 \%$ and $40 \%$, and the HRs of OS are 0.46 and 0.57, respectively [24-30]. We used these assumptions and incorporated status of IDH1 and MGMT promotor into multi-variate Cox regression model. Regarding extent of resection, we believed that subtotal resection is correlated with tumor size. Hence, surgical treatment codes according to tumor size were analyzed to evaluate the impact of margin status on our findings. The same conclusion was drawn by the sensitivity analyses (Additional Table 2). The detrimental effects of AED overall and of valproic acid still existed with adjustment for IDH1, MGMT, or tumor size.

\section{Conclusions}

In summary, OS was not improved and even worse in all patients with AEDs during RT/TMZ in this nationwide population-based study. In terms of individual drugs, VPA is discouraged especially in male and those younger than 65 years old. This topic is highly relevant and merits further study since clinical results were ambiguous. Our real-world data did not support prophylactic use of AED during CCRT, and suggested the potential effect of a specific drug might be distinct in different patient groups.

\section{Abbreviations}

GBM: glioblastoma multiforme, RT: radiotherapy, TMZ: temozolomide, AEDs: anti-epileptic drugs, VPA: valproic acid, OS: overall survival, PFS: progression free survival, CCRT: chemo-radiotherapy, NHIRDs: National Health Insurance research databases, LEV: 
levetiracetam, CCl: Charlson comorbidity index, HRs: hazard ratios, Cls: confidence intervals, HDAC: histone deacetylase, MGMT: 06-methylguanine-DNA methyltransferase

\section{Declarations}

Ethics approval and consent to participate: The research ethics committee of China Medical University and Hospital in Taiwan approved this study (CMUH107-REC3-047).

Consent for publication: Not applicable

Availability of data and materials: Raw data were generated at the National Health Insurance Research Database (NHIRD) published by Taiwan National Health Insurance $(\mathrm{NHI})$ Bureau. Due to legal restrictions imposed by the government of Taiwan in relation to the "Personal Information Protection Act", data cannot be made publicly available. Requests for data can be sent as a formal proposal to the NHIRD (http://nhird.nhri.org.tw). Derived data supporting the findings of this study are available from the corresponding author, Ti-Hao Wang, on reasonable request.

Competing interests: The authors declare that they have no competing interests.

Funding: There was no funding.

Authors' contributions: PYL: Conceptualization, Methodology, Validation, Investigation, Writing - Original Draft, Visualization; KSCC: Resources, Supervision, Funding acquisition; YTW: Conceptualization, Methodology, Validation; CNC: Conceptualization; HCC: Conceptualization; WHC: Software, Formal analysis; THW: Conceptualization, Methodology, Validation, Writing - Review \& Editing, Project administration. All authors read and approved the final manuscript.

Acknowledgments: This research is partially supported by China Medical University Hospital (CMUH) Grant DMR-108-055 and DMR-107-151, and China Medical University Beigang Hospital (CMUBH) Grant 1-CMUBHR108-005

\section{References}

1. Walker MD, Alexander E Jr, Hunt WE, MacCarty CS, Mahaley MS Jr, Mealey J Jr, Norrell HA, Owens G, Ransohoff J, Wilson $\mathrm{CB}$, et al. Evaluation of $\mathrm{BCNU}$ and/or radiotherapy in the treatment of anaplastic gliomas. A cooperative clinical trial. J Neurosurg. 1978;49(3):333-43.

2. Walker MD, Green SB, Byar DP, Alexander E Jr, Batzdorf U, Brooks WH, Hunt WE, MacCarty CS, Mahaley MS Jr, Mealey J Jr, et al. Randomized comparisons of radiotherapy and nitrosoureas for the treatment of malignant glioma after surgery. $\mathrm{N}$ Engl $\mathrm{J}$ Med. 1980;303(23):1323-9.

3. Kristiansen K, Hagen S, Kollevold T, Torvik A, Holme I, Nesbakken R, Hatlevoll R, Lindgren M, Brun A, Lindgren S, et al. Combined modality therapy of operated astrocytomas grade III and IV. Confirmation of the value of postoperative irradiation and lack of potentiation of bleomycin on survival time: a prospective multicenter trial of the Scandinavian Glioblastoma Study Group. Cancer. 1981;47(4):649-52.

4. Shapiro WR, Green SB, Burger PC, Mahaley MS Jr, Selker RG, VanGilder JC, Robertson JT, Ransohoff J, Mealey J Jr, Strike TA, et al. Randomized trial of three chemotherapy regimens and two radiotherapy regimens and two radiotherapy regimens in postoperative treatment of malignant glioma. Brain Tumor Cooperative Group Trial 8001. J Neurosurg. 1989;71(1):1-9.

5. Hochberg FH, Pruitt A. Assumptions in the radiotherapy of glioblastoma. Neurology. 1980;30(9):907-11.

6. Kelly PJ, Daumas-Duport C, Kispert DB, Kall BA, Scheithauer BW, Illig JJ. Imaging-based stereotaxic serial biopsies in untreated intracranial glial neoplasms. J Neurosurg. 1987;66(6):865-74.

7. Stupp R, Mason WP, van den Bent MJ, Weller M, Fisher B, Taphoorn MJ, Belanger K, Brandes AA, Marosi C, Bogdahn U, et al. Radiotherapy plus concomitant and adjuvant temozolomide for glioblastoma. N Engl J Med. 2005;352(10):987-96.

8. Stupp R, Hegi ME, Mason WP, van den Bent MJ, Taphoorn MJ, Janzer RC, Ludwin SK, Allgeier A, Fisher B, Belanger K, et al. Effects of radiotherapy with concomitant and adjuvant temozolomide versus radiotherapy alone on survival in glioblastoma 
in a randomised phase III study: 5-year analysis of the EORTC-NCIC trial. Lancet Oncol. 2009;10(5):459-66.

9. Chie EK, Shin JH, Kim JH, Kim HJ, Kim IA, Kim IH. In Vitro and In Vivo Radiosensitizing Effect of Valproic Acid on Fractionated Irradiation. Cancer Res Treat. 2015;47(3):527-33.

10. Thotala D, Karvas RM, Engelbach JA, Garbow JR, Hallahan AN, DeWees TA, Laszlo A, Hallahan DE. Valproic acid enhances the efficacy of radiation therapy by protecting normal hippocampal neurons and sensitizing malignant glioblastoma cells. Oncotarget. 2015;6(33):35004-22.

11. Eckert M, Klumpp L, Huber SM. Cellular Effects of the Antiepileptic Drug Valproic Acid in Glioblastoma. Cell Physiol Biochem. 2017;44(4):1591-605.

12. Krauze AV, Myrehaug SD, Chang MG, Holdford DJ, Smith S, Shih J, Tofilon PJ, Fine HA, Camphausen K. A Phase 2 Study of Concurrent Radiation Therapy, Temozolomide, and the Histone Deacetylase Inhibitor Valproic Acid for Patients With Glioblastoma. Int J Radiat Oncol Biol Phys. 2015;92(5):986-92.

13. Happold C, Gorlia T, Chinot O, Gilbert MR, Nabors LB, Wick W, Pugh SL, Hegi M, Cloughesy T, Roth P, et al. Does Valproic Acid or Levetiracetam Improve Survival in Glioblastoma? A Pooled Analysis of Prospective Clinical Trials in Newly Diagnosed Glioblastoma. J Clin Oncol. 2016;34(7):731-9.

14. Barker CA, Bishop AJ, Chang M, Beal K, Chan TA. Valproic acid use during radiation therapy for glioblastoma associated with improved survival. Int J Radiat Oncol Biol Phys. 2013;86(3):504-9.

15. Weller M, Gorlia T, Cairncross JG, van den Bent MJ, Mason W, Belanger K, Brandes AA, Bogdahn U, Macdonald DR, Forsyth P, et al. Prolonged survival with valproic acid use in the EORTC/NCIC temozolomide trial for glioblastoma. Neurology. 2011;77(12):1156-64.

16. Ryu JY, Min KL, Chang MJ. Effect of anti-epileptic drugs on the survival of patients with glioblastoma multiforme: A retrospective, single-center study. PLoS One. 2019;14(12):e0225599.

17. Li Z, Xia Y, Bu X, Yang D, Yuan Y, Guo X, Zhang G, Wang Z, Jiao J. Effects of valproic acid on the susceptibility of human glioma stem cells for TMZ and ACNU. Oncol Lett. 2018;15(6):9877-83.

18. Berendsen S, Varkila M, Kroonen J, Seute T, Snijders TJ, Kauw F, Spliet WG, Willems M, Poulet C, Broekman ML, et al. Prognostic relevance of epilepsy at presentation in glioblastoma patients. Neuro Oncol. 2016;18(5):700-6.

19. Ozbek N, Cakir S, Gursel B, Meydan D. Prognostic significance of seizure in patients with glioblastoma multiforme. Neurol India. 2004;52(1):76-8.

20. Liang J, Lv X, Lu C, Ye X, Chen X, Fu J, Luo C, Zhao Y. Prognostic factors of patients with Gliomas - an analysis on 335 patients with Glioblastoma and other forms of Gliomas. BMC Cancer. 2020;20(1):35.

21. Dobran M, Nasi D, Chiriatti S, Gladi M, Somma LD, lacoangeli M, Scerrati M: Prognostic Factors in Glioblastoma: Is There a Role for Epilepsy? Neurol Med Chir (Tokyo) 2018, 58(3):110-115.

22. Glantz MJ, Cole BF, Forsyth PA, Recht LD, Wen PY, Chamberlain MC, Grossman SA, Cairncross JG. Practice parameter: anticonvulsant prophylaxis in patients with newly diagnosed brain tumors. Report of the Quality Standards Subcommittee of the American Academy of Neurology. Neurology. 2000;54(10):1886-93.

23. Perry J, Zinman L, Chambers A, Spithoff K, Lloyd N, Laperriere N. The use of prophylactic anticonvulsants in patients with brain tumours-a systematic review. Curr Oncol. 2006;13(6):222-9.

24. Yang P, Zhang W, Wang Y, Peng X, Chen B, Qiu X, Li G, Li S, Wu C, Yao K, et al. IDH mutation and MGMT promoter methylation in glioblastoma: results of a prospective registry. Oncotarget. 2015;6(38):40896-906.

25. Yan W, Zhang W, You G, Bao Z, Wang Y, Liu Y, Kang C, You Y, Wang L, Jiang T. Correlation of IDH1 mutation with clinicopathologic factors and prognosis in primary glioblastoma: a report of 118 patients from China. PLoS One. 2012;7(1):e30339.

26. Hu WM, Wang F, Xi SY, Zhang X, Lai JP, Wu HY, Liu LL, Sai K, Zeng J. Practice of the New Integrated Molecular Diagnostics in Gliomas: Experiences and New Findings in a Single Chinese Center. J Cancer. 2020;11(6):1371-82.

27. Kim YS, Kim SH, Cho J, Kim JW, Chang JH, Kim DS, Lee KS, Suh CO. MGMT gene promoter methylation as a potent prognostic factor in glioblastoma treated with temozolomide-based chemoradiotherapy: a single-institution study. Int J Radiat Oncol Biol Phys. 2012;84(3):661-7. 
28. Yang H, Wei D, Yang K, Tang W, Luo Y, Zhang J. The prognosis of MGMT promoter methylation in glioblastoma patients of different race: a meta-analysis. Neurochem Res. 2014;39(12):2277-87.

29. Chen Y, Hu F, Zhou Y, Chen W, Shao H, Zhang Y. MGMT promoter methylation and glioblastoma prognosis: a systematic review and meta-analysis. Arch Med Res. 2013;44(4):281-90.

30. Xia L, Wu B, Fu Z, Feng F, Qiao E, Li Q, Sun C, Ge M. Prognostic role of IDH mutations in gliomas: a meta-analysis of 55 observational studies. Oncotarget. 2015;6(19):17354-65.

\section{Figures}
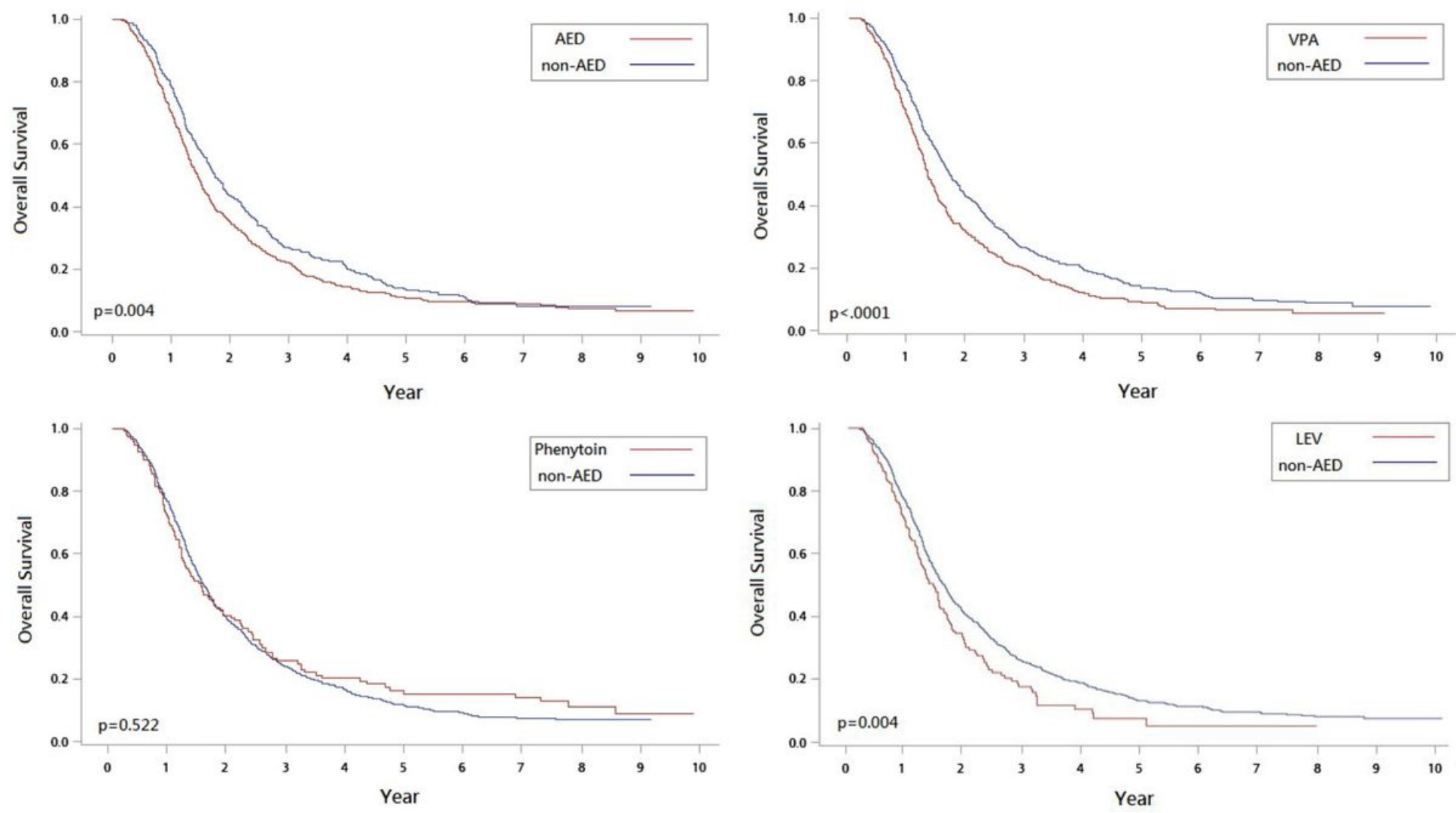

Figure 1

Kaplan-Meier curves of survival in patients with all types of AEDs (A), with VPA (B), with LEV (C), and with phenytoin (D) compared to non-AED group. Abbreviations: AED=anti-epileptic drugs; VPA=valproic acid; LEV=levetiracetam

\section{Supplementary Files}

This is a list of supplementary files associated with this preprint. Click to download.

- Additionalfiles.docx 